\title{
Modeling and Reorganizing of Process Chains Using CIMOSA
}

\author{
Barbara Janusz \\ Institute for Real-Time Computer Systems and Robotics, University of \\ Karlsruhe, D-76128 Karlsruhe, Germany \\ Phone: ++49 721608 4264, Fax: ++49 721606 740, \\ e-mail: bjanusz@ira.uka.de
}

\begin{abstract}
Enterprise models are used in enterprises for several planning tasks, e.g., redesigning of processes or organizational structures, documentation of processes, planning of the computer usage or software development. For the process redesigning, we use the CIMOSA modeling framework. However, many of the existing CIMOSA models of particular enterprises are not complete, not consistent and not optimal. Also, they often describe only functions or subprocesses limited by department borders of an enterprise.

In order to overcome these problems, we developed a new algorithm. It filters out processes of an existing CIMOSA model of an enterprise. These processes all serve to achieve a particular goal. Using the algorithm, also the completeness and the consistency of the considered processes can be checked. An extension of the algorithm serves for the process optimization .
\end{abstract}

Keywords

CIMOSA, enterprise modeling, process redesigning, process optimization

\section{INTRODUCTION}

\subsection{Enterprise modeling}

In an enterprise, several organizational tasks come up, for example the redesigning of business processes and of organizational structures or the documentation according to ISO 9000 . Other examples are planning tasks like planning of the computer usage and the enterprise wide integration of data, optimal usage of resources, the development of control software or the design of new processes. 
In order to solve tasks of this kind, a detailed description of the relevant aspects of the enterprise, an enterprise model, is needed. The most important aim of such a model is to provide a detailed and well structured overview of what happens in the enterprise. Using an enterprise model, also inconsistencies in processes can be recognized and their consequences can be analyzed.

\subsection{Requirements on enterprise models}

Vernadat (1995) presents several criteria which should be satisfied by enterprise models, e.g.:

- generality and reusability:

The model generation is a long and expensive process. Hence, a universal model of an enterprise should exist, which can be reused for each arbitrary task.

- efficiency:

The model should efficiently support the problem solving process, i.e., it should only contain the relevant information for the performance of a particular task. Otherwise, the model becomes too complex and too confused. The efficiency and the generality requirements come into conflict with each other. In this paper, a possibility for solving this conflict is shown.

- extensibility:

Each enterprise model should be able to be expanded.

- consistency and completeness:

These are essential properties of models. It is difficult to guarantee model consistency and completeness, especially in case of multiple users.

- process orientation:

In an enterprise, two components are distinguished: functions (or activities) and processes. The main emphasis of a functional consideration of an enterprise is the description of the task scope of a work place or of a department (Katzy (1995)). Since the model should provide an overview of the enterprise, beside single activities whole processes should be modeled (AMICE (1993)). A process can be partitioned recursively into subprocesses and activities. All subprocesses and activities belonging to a process consume, use or produce objects which are dependent or based on each other. Their execution leads to a common goal (Schaefer (1989)).

- optimality:

The modeled processes should be as straightforward and as optimal as possible.

In the following (section 2), it is shown that the CIMOSA models of particular enterprises do not necessarily satisfy these requirements although the CIMOSA modeling framework provide some concepts which help to achieve this goal. In section 3, an approach for overcoming this problem is presented. Its evaluation is the content of section 4. The paper finishes with an overview on the present and future work concerning this approach.

\section{THE MODELING CONCEPTS OF CIMOSA}

\subsection{CIMOSA modeling framework}

This paper is based on the enterprise modeling concept CIMOSA (Open System Architecture for CIM). A comprehensive overview of and a comparison between the CIMOSA concept and other enterprise modeling concepts can be found in CEN (1994) and Naeger (1995). A detailed 
description of the modeling framework can be found in AMICE (1993). Therefore, in this paper CIMOSA is introduced only shortly.

CIMOSA consists of an Enterprise Modeling Framework and an Integrating Infrastructure. In the following, only the modeling framework is considered. In this framework, the enterprise model is generated using the following three dimensions:

- Instantiation which is the way to create a model of a particular enterprise using the Generic Building Blocks and Partial Models.

- Derivation which describes the modeling process in three stages: the Requirements Definition Modeling, the Design Specification Modeling, and the Implementation Description Modeling.

- Generation, whereby four views on an enterprise are defined: the Function View, the Information View, the Resource View and the Organization View.

\subsection{Modeling according to CIMOSA}

The CIMOSA concept provides a user guide for the generation of particular enterprise models (AMICE (1993) and AMICE (1994)). This guide only generally describes the modeling process by defining the steps to be carried out. Therefore, the user does not get a real support from this guide. Some existing CIMOSA enterprise models of particular enterprises, e.g., Zelm (1995), Neuscheler (1995), Hohn (1993), Schlotz (1995) and the CIMOSA user guide were considered and compared with the requirements presented in section 1.2. Following results were obtained:

- generality, reusability and efficiency:

The models were generated for a specific task, e.g., the design of a logistic system (Schlotz (1995)) or optimization over time in the production (Zelm (1995)). For each other problem, a new model of the enterprise must be generated, because the existing models are strongly limited and hence not universal enough to be reused without an adaptation to the new problem.

- extensibility, completeness and consistency:

The user guide provides a consistency check at the end of the modeling process without proposing any methods for consistency check. Also, the completeness of a model is not ensured. Therefore, a fault free extensibility method does not exist for CIMOSA models.

- process orientation:

According to AMICE (1994) and Vernadat (1995) domains must not be confused with organizational departments. They should be logical groupings of processes with the aim to simplify the enterprise. But in the user guide, no methods are proposed which could clarify how to get a process oriented model. Therefore, often, a process is represented by several subprocesses and the connections between them are not obvious immediately. Also, some activities belong to the modeled processes, which lead to other goals than the considered ones.

- optimality:

In CIMOSA concept, no methods for the optimization of processes are provided.

\section{RESTRUCTURING OF CIMOSA MODELS}

Summarized, the CIMOSA concept ensures the satisfaction of only few of the requirements presented in section 1.2. The user guide is not strict enough to restrict the user to create a unique model of an enterprise which satisfies the requirements. In principal, some concepts are provided in CIMOSA which should support the meeting of the requirements. But there is no 
methodology which could limit the modeling process and force the user to create a model which meets the requirements.

In order to overcome this problem, the following approach is proposed: It is assumed, that a CIMOSA model of an enterprise describing the as-is situation exists. It contains several information about the enterprise. Possibly, the model is not process oriented or the processes are limited by departments borders. Using the following approach, such an arbitrarily structured model can be restructured in order to achieve processes leading to some specific objectives and to better satisfy the requirements on enterprise models. Beside this, an optimal to-be situation is described, which can be realized in the enterprise afterwards. The approach consists of two steps:

1. Reorganizing of the given CIMOSA model in order to get task specific processes.

2. Optimizing the restructured processes.

For the first step, an algorithm has been developed which filters the needed information out of a general CIMOSA model of a particular enterprise (section 3.1). This information forms a process which leads to a given goal. Using the algorithm, incomplete or inconsistent parts of the enterprise model can be detected. In a further step, the reconstructed process can be additionally optimized (section 3.2).

\subsection{Forming processes}

\section{Modeling activities with CIMOSA}

Using CIMOSA, activities are modeled by the object Enterprise Activity (see Figure 1).

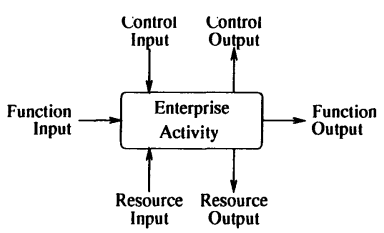

Figure 1 The CIMOSA object Enterprise Activity.

By definition, an Enterprise Activity transforms its Function Input into the Function Output using its Control and Resource Inputs (AMICE (1994)). Possibly, a Control Output and a Resource Output can be produced. Hence, an Enterprise Activity is defined by its functionality, i.e., its Function, Control and Resource Inputs and Outputs. The Enterprise Activity is a central construct for the algorithm presented in the following. The description of Enterprise Activities on the Requirements Definition Modeling Level can be used, because no resource issues are considered in this paper.

\section{The process algorithm}

Because the algorithm is used for forming processes which are dedicated to achieve a given goal, the algorithm works backwards from the goal of a process to ist first activity:

1. Define the desired output objects of the process. 
2. Look for all activities producing these objects as their Function Output. They belong to the last stage of the process.

3. Define the objects which are used by these activities as their Function or Control Input.

4. Look for all activities producing these objects as their Function Output. They are predecessors of the considered activities.

5. Finish, if all currently considered activities do not have any inputs or if they only need inputs which are assumed to be given, otherwise go back to step 3 .

The result of the algorithm is a precedence graph which gives the sequence of the execution of activities needed in order to achieve a given goal.

\section{Example: Forming a process}

\section{The modeled enterprise:}

In order to show the functionality of the process algorithm, a simplified example is considered. In this example, two departments of the enterprise have already been modeled: the inventory and the production department.

- The inventory department:

The inventory department purchases raw material and receives it (Figure 2). After an inspection of the raw material, its vendor is rated and the decision about using it for manufacturing or reordering it because of material faults is taken.

\section{Inventory:}

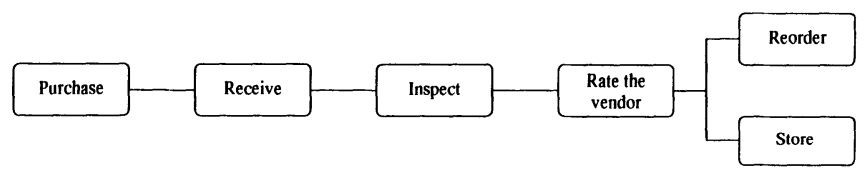

Figure 2 The activities of the inventory department.

- The production department:

The production starts with some preparations like getting the raw material and tools and loading the NC program (Figure 3). Afterwards, the raw material is processed in order to manufacture the product. If the raw material is not used completely, the decision about the utilization of the remaining amount must be taken.

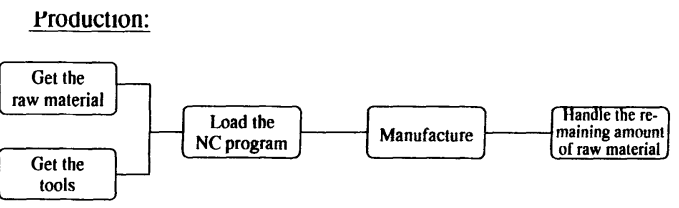

Figure 3 The activities of the production department. 


\section{The process:}

The user's interest is the creation of a product. Therefore, he wishes a view on the modeled enterprise, where all needed activities belong to a process „Product creation“ and where the irrelevant activities do not occur.

Figure 4 shows the pool of CIMOSA Enterprise Activities describing the enterprise. In order to find the last activity of the restructured process, the algorithm looks for all activities whose Function Output is the manufactured product. In the example, it is the activity „Manufacture“. Now, the inputs of this activity must be considered: the predecessor activities must deliver the raw material and the reports on the completion of the tools and machine setups. This procedure repeats until the activity „Purchase“ is found, because this activity does not need any inputs. The complete process is shown in Figure 5. All activities in this process participate in achieving the goal „Product creation“ and no irrelevant activities belong to it (e.g., „Rate the vendor“).

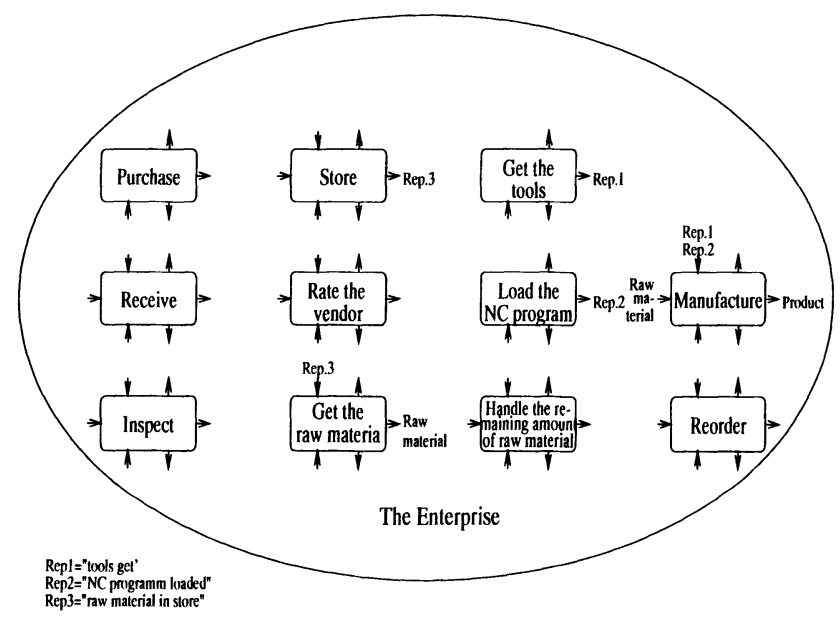

Figure 4 Set of Enterprise Activities in the modelled enterprise (incomplete).

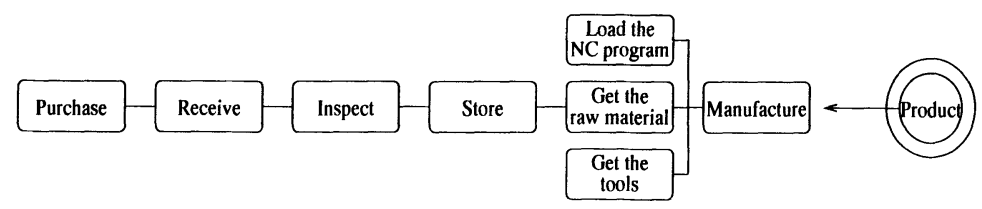

Figure 5 The restructured process .

\section{Completeness and consistency of the process}

The inputs and outputs of the Enterprise Activities are pointers to other CIMOSA views. Therefore, by guaranteeing the completeness of the Enterprise Activities in the process and by assuming that the inputs and the outputs of an Enterprise Activity are described, the completeness of a process is ensured when all Enterprise Activities belonging to it are described in the model. 
Using the algorithm described above, the places in the process where information is missing are indicated to the user. This is always the case, when the algorithm cannot find an Enterprise Activity which produces the Function Output needed as the Function or Control Input by other activities belonging to the process. Hence the user gets detailed indications to information items which are missing.

Concerning the consistency of the modeled information, the algorithm can point out the places, where the consistency of the Function View is not guaranteed. It does not find out inconsistencies in the other views.

Using the algorithm, the user is referred to the points, where inconsistencies can occur. There are several possibilities for inconsistencies:

- different processes which achieve the same goal, i.e., which have the same output,

- different goals for a single process,

- processes with interchangeable Enterprise Activities.

These cases can indicate inconsistencies but they do not do it necessarily. Therefore, the inconsistencies cannot be removed automatically. The decision about possible further steps for removing them must be taken by the user.

\subsection{Optimization of the processes}

In order to form the processes, only the Function and the Information View are considered. Therefore, the result of the algorithm is a precedence graph which gives all possibilities for paralleling and all places, where the activities must be executed sequentially. The Resource and the Organization View are not considered up to now.

It is necessary to investigate the used and the given resources because the feasibility of a parallel execution of activities is only ensured if all needed resources are available. Also the authority and responsibility fields of some organizational units must be considered in order to investigate eventual conflicts while allocating the resources and objects needed for some activities. Beside this, weak points in a process should be detected. Therefore, the optimization of a process is carried out in three steps:

1. feasibility analysis,

2. weak points analysis,

3. evaluation of alternative processes and selection of the optimal process.

Because for these purposes the descriptions of resources and alternative processes are needed, also the Design Specification Modeling Level must be investigated.

\section{Feasibility analysis}

In the first step, the feasibility analysis, the availability of the needed resources is checked. Up to now, only the static availability for single processes is investigated using the Resource View. The interrelation of several processes at run time is not considered yet.

\section{Weak points analysis}

The second step, the analysis of possible weak points in a process, consists of several sub tasks (see, e.g., Ferstl (1995)):

1. investigation of breaks in the media chains:

The media type (readable by machines, by humans or by both of them) should be the same for all information processing activities in the process. Only then problems of multiple data 
capture and redundancy, or consistency problems can be avoided. Using the information stored in the Resource View, breaks in media chains can be found. It is the task of the user to decide, how these breaks can be avoided.

2. investigation of changes of the degree of automation:

For the activities processing physical objects, the degree of automation is investigated. The goal is to get chains with a unique automation degree (fully or partial automated or manual), because only then time, cost and quality losses can be avoided. In analogy to point 1 , the Resource View is investigated for this purpose.

3. investigation of the organizational assignments:

Only if all activities and the appropriate resources and objects belong to one organizational unit, conflicts can be avoided. Then, the coordination expenses and the degree of information exchanges are minimized. Beside this, the organizational unit has an overview of the whole process. In order to detect weak organizational points in a process, the authorities and responsibilities for each activity and the corresponding resources and objects must be investigated. Therefore, for this task all four CIMOSA views are considered.

4. investigation of the assignment of resources:

For an optimal utilization of resources, they should be released for their reuse as soon as they are no more required for the execution of an activity. Problems can arise if some sets of resources are reserved for the whole duration of an activity and only a part of these resources are needed all the time. In order to find out which resources can be released earlier, the process and the corresponding information stored in the Resource View must be considered.

Beside these possibilities, several other aspects should be investigated. For detailed description of further possible weak points, see, e.g., Ferstl (1995).

\section{Evaluation of the alternative processes}

Performing the analysis presented in the previous section, the weak points of a process can be detected but they cannot be removed automatically. Therefore, the user decides how he can solve these problems. In most cases, he will develop several alternative solutions, hence his result will be a set of alternative processes. These alternative processes can be evaluated according to several user defined features like time, cost or quality. For this purpose, an evaluation algorithm was developed by Neuscheler (1994) as an extension of the CIMOSA concept. Using this evaluation algorithm, the user can select his optimal process.

\section{EVALUATION OF THE PROPOSED APPROACH}

\subsection{Unsolved problems}

For both, the process algorithm and the optimization algorithm, some problems must be solved before implementation. They will be shortly presented in the following.

\section{Process algorithm}

Consider the example in Figure 6 and assume that we look for a process which creates product 03. The result of the algorithm is a set of several possible processes (Figure 7). Difficulties of this kind are due to the only consideration of the inputs and outputs of activities. The semantics of activities and objects and the necessary sequences of the execution of activities cannot be recognized if there are activities with the same inputs and outputs. In order to overcome these 
problems, different solutions must be investigated before a solution can be found. Presently, the three following alternatives are being considered:

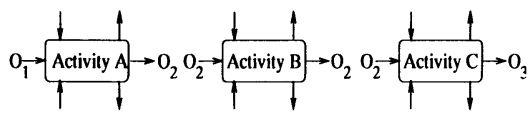

Figure 6 An activity pool.

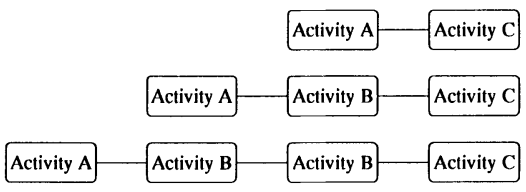

Figure 7 The precedence graph.

1. marking the processing state of used objects:

Using particular entries in the object description, it would be easy to recognize the necessary sequence of the execution of activities in the process.

2. investigation of the alternative process es:

In this case, the all alternative processes must be investigated, e.g., processess with loops or with different lengths. A solution could be to accept only processes without loops and to choose always the longest process for further investigations in order to ensure that all needed activities are modeled in the process.

3. classification of the activities:

For this purpose, a classification similar to the one presented by Vernadat (1995) is investigated. It is extended by introducing particular properties of function and control inputs and outputs which must be set if the corresponding activity is of a given type. Then, additional rules depending on these properties and types can be used for the process algorithm.

\section{Optimization algorithm}

The problem of all optimization algorithms is the semantics. Up to now, only optimization approaches can be formalized and implemented which are independent of the semantics of activities or objects. If the semantics cannot be negliged, a method for the classification of activities like presented in Vernadat (1995) can be used.

\subsection{The advantages of the approach}

With the algorithms presented in section 3 , the requirements posed on enterprise models (see section 1.2) can be fulfilled without forcing the user to create a particular model:

- generality, reusability and efficiency:

An enterprise model describing a wide part of the enterprise can be reused for different tasks. For each new task, the appropriate information is filtered out of the model using the algorithm presented in section 3.1. The resulted model is as efficient as possible.

- extensibility, completeness and consistency:

New domains or processes can be added to the model. The corresponding Enterprise Activities will be considered automatically at the next time the process algorithm is executed. The completeness and the consistency are then also checked (section 3.1).

- process orientation:

The result of the process algorithm is a process crossing several department borders. Its activities use, consume or produce common information to achieve a common goal. 
- optimality:

The modeled processes are simplified, because they only contain these activities and information which are needed for achieving a given goal. The optimization process is supported by the analysis described in section 3.2 .

\section{CONCLUSIONS AND FUTURE WORK}

In this paper, a new approach is presented which helps to satisfy the requirements posed on enterprise models. By filtering task specific processes out of a more general and comprehensive enterprise model, a process oriented model is achieved which only contains the relevant information for the solution of a given task. The processes are optimized using the approach presented in section 3.2. An additional advantage of this approach is, that the user does not need to have a global view on the entire enterprise to generate the models of various processes. Each department or even smaller part of an enterprise can be modeled separately. Only the interfaces between the parts must be clearly defined. The explicit connections between activities in the departments are generated by the process algorithm and thus the process oriented modeling as required in AMICE (1994) is achieved.

Presently, the solution for the difficulties presented in section 4 are being worked out and the approach is being implemented.

Up to now, only single processes are considered. As a next step, the correlation between several processes and a common optimization, e.g., the check of availability of resources at the run time, will be investigated.

Also, possible further applications of the proposed approaches will be investigated. There are different possibilities for the utilization of processes:

- consistency check:

For the consistency check of a particular enterprise model, processes with the appropriate goals are formed. For ensuring consistency of the originally modeled processes, they must be compared with the formed precedence graph.

- benchmark tests:

In order to compare several enterprises and their performance, processes with a common goal are formed for each of the enterprises considered. Using the evaluation algorithm presented by Neuscheler (1994) they can be evaluated objectively.

- planning tasks:

There are several publications, e.g., Naeger (1994) and Schaefer (1989), describing applications for processes. Using the algorithm presented above, these processes can be computed instead of assuming that they are given.

Also the second part of the presented approach, the optimization algorithm, can be used for other purposes. Several ideas exist for extensions of this algorithm in order

- to implement business process redesigning approaches which are more comprehensive than the algorithm presented in this paper,

- to model organizational restructuring approaches,

- to model some new enterprise paradigms like fractal enterprise (Warnecke (1993)) or holonic enterprise (Seidel (1994)). 


\section{ACKNOWLEDGMENTS}

This research was performed at the Institute for Real-Time Compter Systems and Robotics (IPR), Prof.Dr.-Ing. U. Rembold and Prof.Dr.-Ing. R. Dillmann, Computer Science Department, University of Karlsruhe. We would like to thank Prof. Rembold for his support.

\section{REFERENCES}

ESPRIT Consortium AMICE (1993) CIMOSA: Open System Architecture for CIM. SpringerVerlag.

ESPRIT Consortium AMICE (1994) Formal Reference Base - ESPRIT Project 7110 AMICECIMOSA, M-3 Deliverables - Volume 3.

Ferstl, O.K. and Sinz, E.J. (1995) Re-engineering von Geschaeftsprozessen auf der Grundlage des SOM-Ansatzes. Bamberger Beitraege zur Wirtschaftsinformatik, 26.

Hammer, M. and Champy, J. (1993) Reengineering the Corporation. Harper Collins.

Hohn, Th. (1993) Vergleich von Unternehmmensmodellen auf der Basis offener Systemarchitekturen mit einem objektorientierten Modellierungsansatz am Beispiel eines Sondermaschinenherstellers. Master's thesis, University of Karlsruhe.

Katzy, B.R. (1995) Unternehmensplanung in produzierenden Unternehmen. Verlag Shaker, Aachen.

Naeger, G. (1994) An Integrated Approach to Software Systems Planning and Selection Based on CIMOSA Models.Concurrent Engineering: Research and Applications.

Naeger, G. and Reithofer, W. (1995) Bottom-up Planning Approach in Enterprise Modelling The Need and the State of the Art. CIM at Work Conference Eindhoven.

NAM (1991) Rechnerintegrierte Fertigung: Systemarchitektur - Rahmenwerk zur Unternehmensmodellierung.

Neuscheler, F. and Spath, D. (1994) The Economic View: A Concept Using Benchmarks to Analyze, Evaluate and Optimize Business Processes. Proceedings of the IMSE'94 Conference, Grenoble.

N.N. (1994) An evaluation of CIM modelling constructs - Evaluation report of constructs for views according to ENV 40003.Computers in Industry, 24, 159-236.

Schaefer, H. (1989) CAD / CAM: Planung langfristiger Gesamtkonzeptionen. VDI Verlag, Duesseldorf.

Schlotz, C. and Roeck, M. (1995) Reorganization of a Production Department According to the CIMOSA Concepts.Computers in Industry, 27:179-189.

Seidel, D. (1994) HMS-Strategies, Overview. IMS - Holonic Manufacturing Systems: Deliverable WP6.

Vernadat, F.B. (1995) Busines Process and Enterprise Activity Modelling. The 11th ISPE/ IEE/IFAC International Conference on CAD/CAM Robotics and Factories of the Future' 95. Columbia.

Vernadat, F.B. (1995) CIM Business Process Modeling and Analysis. The 11th ISPE/IEE/ IFAC International Conference on CAD/CAM Robotics and Factories of the Future'95. Columbia.

Warnecke, H.-J. (1993) Revolution der Unternehmenskultur - Das fraktale Unternehmen. Springer Verlag, Berlin.

Zelm, M., Vernadat, F.B. and Kosanke, K. (1995) The CIMOSA Business Modelling Process. Computers in Industry, 27, 123-42. 


\section{THE AUTHORS}

Barbara Janusz obtained her diploma in computer science in 1994 from the University of Karlsruhe, Germany. Since January 1995, she has been a research assistant at the Institute for Real-Time Computer Systems and Robotics at the University of Karlsruhe. Her main interests are in enterprise modeling, business process redesigning as well as hierarchical management and control of manufacturing systems. 